\title{
Constant-phase element identification in conductivity sensors using a single square wave
}

\author{
Javier Lario-García, Ramon Pallàs-Areny* \\ Instrumentation, Sensors and Interfaces Group, Castelldefels School of Technology (EPSC), Technical University of Catalonia (UPC), \\ Avda. Canal Olímpic s/n, Building C4, 08860 Castelldefels (Barcelona), Spain \\ Received 22 September 2005; received in revised form 14 March 2006; accepted 11 April 2006 \\ Available online 24 May 2006
}

\begin{abstract}
This paper proposes a novel method to measure the electrical conductivity of solutions in the time domain by using a single square-wave current and two titanium electrodes. This method allows us to determine both the conductivity $(\sigma)$ of the solution and the two parameters of the constantphase element (CPE) due to the electrodes. Experimental results show that the proposed method achieves conductivity measurement ranges close to those obtained with platinum probes or tetrapolar measurements, which are more expensive, and provides information about electrode condition. (C) 2006 Elsevier B.V. All rights reserved.
\end{abstract}

Keywords: Conductivity sensor; Constant-phase element; Time-domain measurement

\section{Introduction}

The conductivity of solutions is a very important parameter in many industrial material-manufacturing processes. Conductivity sensors for solutions involve ionic and electronic currents, so they use electrodes that convert ionic currents into electronic currents, and conversely [1]. Industrial applications need conductivity probes based on electrode materials such as stainless steel or titanium, which are more robust than platinum and graphite, common in laboratory measurements. Because of their high impedance, the usual measurement range for those robust electrodes is two orders of magnitude narrower than that for platinum. For this reason, high conductivity $\left(\sigma>30 \mathrm{mS} \mathrm{cm}^{-1}\right)$ industrial probes are normally tetrapolar, hence more expensive than two-electrode probes.

Electrical impedance is normally measured by injecting a sinusoidal stimulus. Square-wave stimuli are easier to generate but need special detection methods unless the measured impedance is a simple resistance. Complex impedances can be measured with square-wave stimuli but most of the reported methods that use them rely on calculations that involve Fourier transforms, hence they are difficult to apply in compact systems.

\footnotetext{
* Corresponding author. Tel.: +34 93413 7096; fax: +34 934137007.

E-mail address: ramon.pallas@upc.edu (R. Pallàs-Areny).
}

Some conductivity measurements in the time domain use triangular signals [2], for example, by analysing the time derivative of the applied voltage or the current through the material, or by applying a step current and measuring the drop in voltage across the electrodes after a few microseconds (transient resistance measurement) [3]. These methods are not analytical, so they do not need to previously model the impedance to be measured. But they do not measure the electrode impedance and therefore cannot provide self-diagnostic capability to the system.

This paper proposes a novel method to measure the electrical conductivity of solutions that uses a single square-wave current and a conductivity cell built from two titanium electrodes, yet achieves a measurement range close to that obtained with platinum electrodes. The proposed method reduces manufacturing costs and allows us to measure electrodes' impedance, which is useful to assess their condition.

\section{Equivalent circuits in conductivity sensors}

Electrode-electrolyte systems are often modelled by lumpedelement circuits [4]. Fig. 1a shows the equivalent circuit for a system that comprises two electrodes and a liquid solution [5], where $Z_{\mathrm{S}}$ is the solution impedance, $R_{\mathrm{F}}$ the Faradic resistance and $Z_{\mathrm{CPE}}$ is the so-called constant-phase element, which models 
(a)

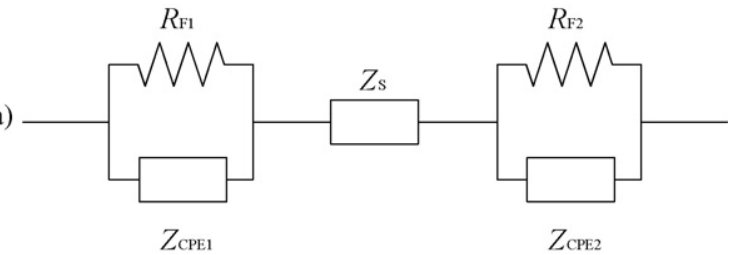

(b)

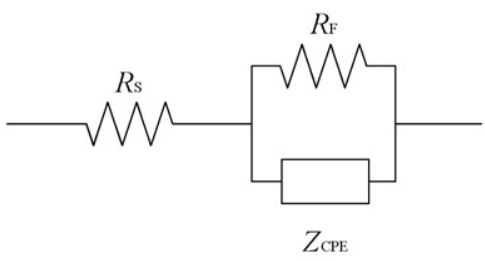

Fig. 1. Equivalent circuit for: (a) an electrolyte measured with a pair of electrodes and (b) a saline solution when the two electrodes are equal.

the electrodes [6],

$Z_{\mathrm{CPE}}=\frac{1}{A s^{\alpha}}$

For saline solutions, $Z_{\mathrm{S}}=R_{\mathrm{S}}$, the electrolyte resistance ([1], Chapter 2), and, if the two electrodes in Fig. 1a have similar characteristics, the equivalent circuit reduces to that shown in Fig. 1b. Therefore, determining those three impedance components would enable us to calculate $R_{\mathrm{S}}$. Fig. 2 shows the complex impedance plot for the impedance of the electrode-electrolyte system for a sample saline solution.

The constant-phase element $Z_{\mathrm{CPE}}$ involves two independent parameters (1), $A$ and the exponent $\alpha(0 \leq \alpha \leq 1)$. When $\alpha=1, A$ has the dimension of a capacitance, otherwise its dimension is $\Omega^{-1} \times s^{-\alpha}(s=\mathrm{j} \omega)$, hence the name pseudocapacitance for $Z_{\mathrm{CPE}}$. When $\alpha=0.5, Z_{\mathrm{CPE}}$ becomes a series $R C$ circuit, often termed Warburg impedance. When $\alpha=0, Z_{\mathrm{CPE}}$ becomes a resistance [7].

Using a four-wire (Kelvin) measurement set-up would overcome the effect of electrode impedance in Fig. 1a ([8], Section 3.1 ), but the two additional electrodes needed make conductivity probes more difficult to manufacture, and add complexity and cost to circuit design.

Impedance can be measured by applying a single squarewave current and then measuring the resulting voltage $\left(V_{1}, V_{2}\right.$ and $V_{3}$ ) at different times (Fig. 3b). $P$ independent impedance

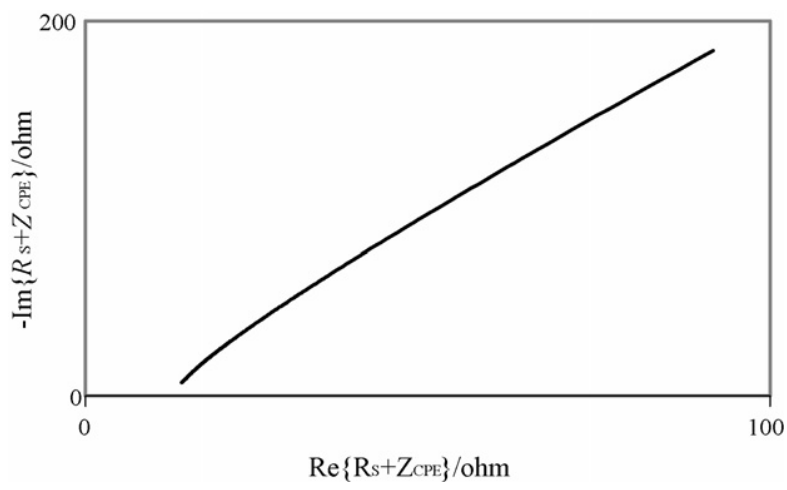

Fig. 2. Complex impedance plot for a system that consists of two electrodes immersed in a saline solution.

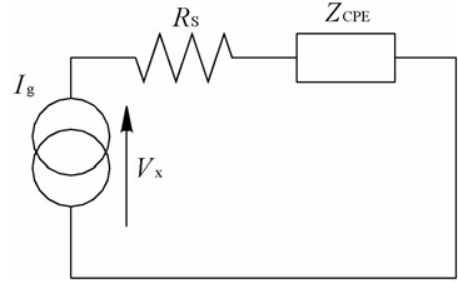

(a)

Fig. 3. (a) Equivalent measurement circuit and (b) voltage measurement at three given times.

components can be determined from at least $P$ independent measurements, as performed in impedance spectroscopy using sine waves [6], or with $P$ current measurements after applying a single square-wave voltage [9]. Here we extend the method developed in [9] for constant-value impedance components, to CPE elements, until now determined from complex impedance diagrams. We apply a single square-wave current (rather than a voltage as in [9]), and measure the resulting voltage at three different times (Fig. $3 \mathrm{~b}$ ). $R_{\mathrm{F}}$ has been determined to be very large for our titanium electrodes and hence its effect is negligible. Solving the equation system consisting of the three voltage amplitudes at the measurement times yields the three independent components. From these components we can determine $R_{\mathrm{S}}$ if the system has been previously calibrated.

Figs. 4 and 5 show that the series combination of the solution resistance plus the constant-phase element $Z_{\mathrm{CPE}}$ is non-linear with the stimulus amplitude. Fig. 4 shows that for sine wave currents of $20 \mathrm{~mA}$ and $0.5 \mathrm{~mA}$, the difference between the measured resistance for each current intensity decreases when the frequency increases. The same happens for the reactance (Fig. 5). Therefore, from Figs. 4 and 5, the non-linear effect of current harmonics is much smaller than that of the fundamental current. Hence, for a square-wave stimulus, if its frequency is high enough and the current density is small enough, non-linearity will be negligible [4].

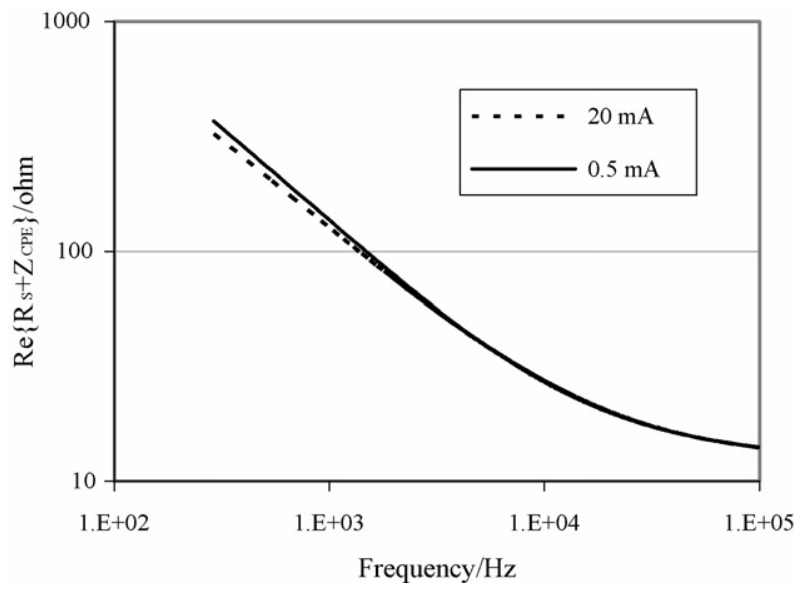

Fig. 4. Real part of the electrode-electrolyte impedance as a function of the frequency for sine wave current intensities of $0.5 \mathrm{~mA}$ and $20 \mathrm{~mA}$. 


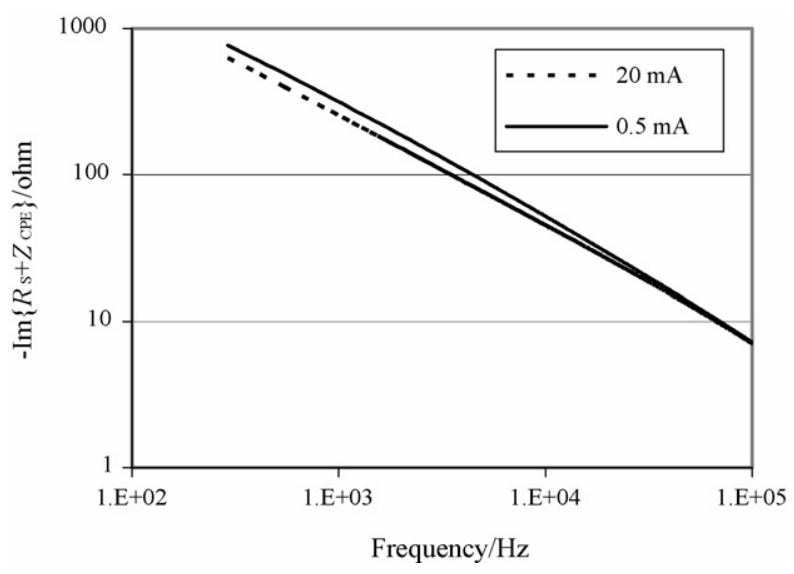

Fig. 5. Imaginary part of the electrode-electrolyte impedance as a function of the frequency for sine wave current intensities of $0.5 \mathrm{~mA}$ and $20 \mathrm{~mA}$.

\section{Circuit analysis}

The overall impedance of the conductivity cell plus electrolyte in Fig. 3a is

$Z(s)=R_{\mathrm{S}}+\frac{1}{A s^{\alpha}}$

The drop in voltage across that impedance when a current step of intensity $I_{0}$ is applied is (see Appendix A)

$v_{\mathrm{U}}=\left[I_{0} R_{\mathrm{S}}+\frac{I_{0}}{A} \frac{t^{\alpha}}{\Gamma(\alpha+1)}\right] u(t)$

where $u(t)$ is the unity step function. Hence, $v_{\mathrm{U}}$ depends on $t^{\alpha}$ rather than $t^{-\alpha}$ as in $[10,11]$, because here we apply current and measure voltage instead of applying voltage and measuring current.

A square-wave current can be decomposed in a sum of current steps of different intensities, each delayed by $T$ seconds, as follows

$i(t)=I_{0}[0.5 u(t)-1.5 u(t-T)+2 u(t-2 T)-2 u(t-3 T)$

$$
+2 u(t-4 T) \cdots]
$$

The application of a square-wave current of intensity $I_{0}$ and period $2 T$ yields a drop in voltage across the impedance

$V_{m}=v(2 M T+m \Delta T)=I_{0} R_{\mathrm{S}}+\frac{I_{0}}{A} \frac{\Delta T^{\alpha}}{\Gamma(\alpha+1)} \Psi_{m}(\alpha, M, N)$

where $M$ is the number of periods elapsed after applying the signal, and

$\Delta T=\frac{T}{N}$

where $N$ is the number of voltage measurements to perform each period, plus 1 (e.g. $N=4$ if we measure three voltages); $m \Delta T$ is the measurement time in the half-period $T, m$ being any integer
$1 \leq m<N$, and

$$
\begin{aligned}
& \Psi_{m}(\alpha, M, N)=0.5(2 M N+m)^{\alpha}-1.5(2 M N-N+m)^{\alpha} \\
& +2(2 M N-2 N+m)^{\alpha}-2 \sum_{k=2}^{M}\{[2(M-k)+1] N+m\}^{\alpha} \\
& +2 \sum_{n=2}^{M}[2(M-n) N+m]^{\alpha}
\end{aligned}
$$

where $\Psi_{m}(\alpha, M, N)$ is the result of the contributions of all previous current steps to the voltages currently being measured.

From the $N-1$ individual voltage measurements we calculate the following voltage ratio:

$\Phi(N)=\frac{V_{1}-V_{2}}{V_{2}-V_{3}}=\frac{v(2 M T+\Delta T)-v(2 M T+2 \Delta T)}{v(2 M T+2 \Delta T)-v(2 M T+3 \Delta T)}$

and from this ratio we obtain $\alpha$ by curve fitting (see Appendix A). For example, if we measure three voltages $V_{1}, V_{2}$ and $V_{3}$ at times $T_{1}, T_{2}$ and $T_{3}$, we have $N=4$, and

$\alpha=0.014954 \times \Phi^{2}(4)-1.5942 \times \Phi(4)+2.4501$

$A$ is obtained from the voltages $V_{m}$ in (5) when $m=1$ and 2,

$A=\frac{I_{0}}{V_{1}-V_{2}} \frac{\Delta T^{\alpha}}{\Gamma(\alpha+1)}\left[\Psi_{1}(\alpha, M, N)-\Psi_{2}(\alpha, M, N)\right]$

From $A$ and $\alpha$, it is straightforward to solve for $R_{\mathrm{S}}$ in (5) when $m=1$.

$R_{\mathrm{S}}=\frac{V_{1}}{I_{0}}-\frac{1}{A} \frac{\Delta T^{\alpha}}{\Gamma(\alpha+1)} \Psi_{1}(\alpha, M, N)$

Finally, from $R_{\mathrm{S}}$ and the cell constant $K$ we obtain the electrolyte conductivity,

$\sigma=\frac{K}{R_{\mathrm{S}}}$

\section{Experimental results and discussion}

The proposed measurement method has been implemented by a custom circuit built on an add-on PC board, and two round titanium electrodes of $5 \mathrm{~mm}$ diameter placed $10 \mathrm{~mm}$ apart with $1 \mathrm{~m}$ cable length. The measurement current was $0.5 \mathrm{~mA}, 1 \mathrm{kHz}$, at low conductivities, and $5 \mathrm{~mA}, 2 \mathrm{kHz}$, at high conductivities (Table 1). The method has been first applied to measure a capacitor, which yielded $\alpha \approx 1$, and $A$ very close to the capacitance value, as expected. Then the method has been applied to six solution samples whose conductivities, in a range from $1 \mathrm{mS} \mathrm{cm}^{-1}$ to $189 \mathrm{mS} \mathrm{cm}^{-1}$, have been determined by a WTW Multi $340 \mathrm{i}$ conductimeter with a TetraCon ${ }^{\circledR} 325$ probe, which uses a fourelectrode graphite conductivity cell.

Geometrical factors in conductivity probes are accounted for by the cell factor $K$ determined as the quotient between the actual solution conductivity and the measured conductance $\left(G_{\mathrm{S}}\right)$,

$K=\frac{\sigma}{G_{\mathrm{S}}}$ 
Table 1

Error for two alternative circuit models for a titanium conductivity sensor for different conductivities measured at a specific current intensity and frequency

\begin{tabular}{|c|c|c|c|c|c|c|}
\hline$\alpha\left(\mathrm{mS} \mathrm{cm}^{-1}\right)$ & $\alpha$ & $A\left(\mu \Omega \times s^{\alpha}\right)^{-1}$ & $I(\mathrm{~mA})$ & $f(\mathrm{kHz})$ & Error for $R$ model (\%) & Error for $R+$ CPE model $(\%)$ \\
\hline 1.0 & 0.7 & 0.07 & 0.5 & 1 & 4.0 & 0.2 \\
\hline 1.9 & 0.74 & 0.15 & 0.5 & 1 & 5.5 & 0.5 \\
\hline 5.5 & 0.81 & 0.51 & 5 & 1 & 17 & 2.9 \\
\hline 42 & 0.92 & 1.37 & 5 & 2 & 39 & 5.7 \\
\hline 93 & 0.92 & 1.39 & 5 & 2 & 61 & 5.6 \\
\hline 189 & 0.92 & 1.77 & 5 & 2 & 74 & 6.4 \\
\hline
\end{tabular}

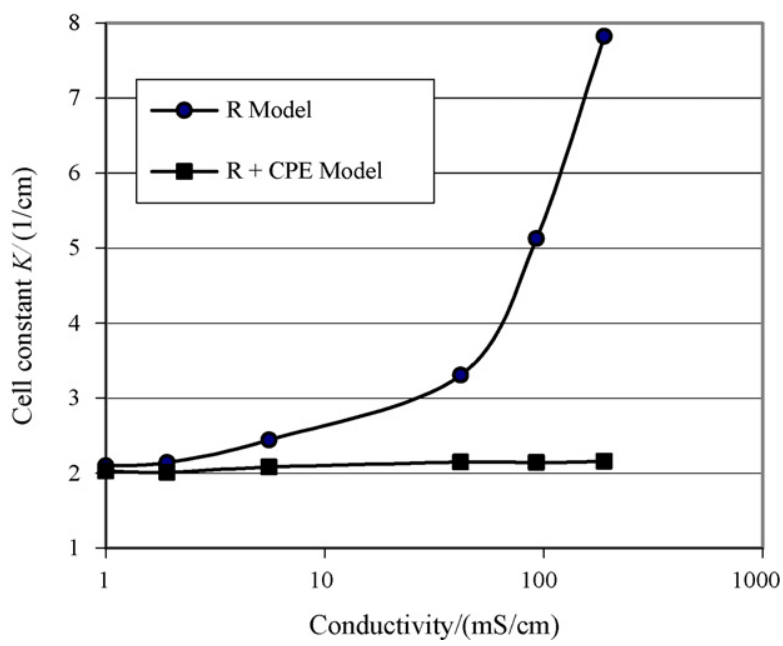

Fig. 6. Dependence of the cell constant $K$ on the conductivity for a titanium probe when modelled by a single $R$ or by $R$ in series with a CPE.

$K$ should depend on geometry only, not on frequency or conductivity. For our titanium probe, by measuring at low conductivities, we determined $K=2.02 \mathrm{~cm}^{-1}$.

Fig. 6 shows that if the cell and electrolyte are modelled as a single resistance $R, K$ increases very much when the electrolyte conductivity increases, which results in an error that limits its measurement range to less than about $3.5 \mathrm{mS} \mathrm{cm}^{-1}$ for accepted errors below $10 \%$. However, if the cell and electrolyte are modelled by a resistance in series with a constant-phase element, the error is less than $7 \%$ along a measurement range which is about two decades wider than that for a simple $R$ model. Table 1 shows measurement errors for both models, for conductivities ranging from $1 \mathrm{mS} \mathrm{cm}^{-1}$ to $189 \mathrm{mS} \mathrm{cm}^{-1}$, and the values for $A$ and $\alpha$, which depend on the conductivity and current density. Were long cables used (say, $10 \mathrm{~m}$ ) and high conductivities measured, cable capacitance $(350 \mathrm{pF})$ would be negligible compared to the impedance of the CPE $\left(\alpha=0.92, A=1.77\left(\mu \Omega \times s^{\alpha}\right)^{-1}\right)$ and $R_{\mathrm{S}}$ $(\sim 10 \Omega)$. Cable inductance $(14 \mu \mathrm{H})$ would also be insignificant because of the low frequency of the injected current. Cable resistance $(\sim 1 \Omega)$, which adds a series resistance to the measured impedance, could become an issue. However, that series resistance can be calibrated out before immersing the probes.

\section{Conclusions}

Titanium conductivity probes based on two electrodes in saline solutions are better modelled by a series resistance for the electrolyte in series with a constant-phase element (CPE) for each electrode. Otherwise, modelling the whole cell by a single resistance yields errors larger than $10 \%$ when measuring conductivities higher than $4 \mathrm{mS} \mathrm{cm}^{-1}$. When a series constant-phase element is included, the two components and three parameters of the model can be determined by solving the equation system formed by the three voltages measured at specific times after injecting a square-wave current into the cell. A custom implementation of this method achieves errors below $7 \%$ for conductivities up to $189 \mathrm{mS} \mathrm{cm}^{-1}$. Therefore, when using the proposed model, the conductivity range for a cell based on two titanium electrodes is about two decades wider than that obtained by a simple resistance model, and the knowledge of the electrode impedance can add self-diagnostic capability to the system by determining the CPE parameters $A$ and $\alpha$.

\section{Acknowledgments}

This project has been partially funded by the Spanish Ministry of Education and Science, contract DPI2002-00707, and the European Regional Development Fund.

\section{Appendix A}

The impedance of the network in Fig. 3a is

$Z(s)=R_{\mathrm{S}}+\frac{1}{A s^{\alpha}}$

The drop in voltage across $Z(s)$ when applying a square-wave current source whose Laplace transform is $I(s)$ is

$V(s)=I(s) Z(s)$

A possible method to obtain the inverse Laplace transform of $V(s)$ is to decompose the square wave of amplitude $I_{0}$ and period $2 T$ into the sum of step signals delayed by $T$ (Fig. 7). The output voltage response of a linear system can be obtained as the addition of the voltages contributed by each input current step. The Laplace transform of a current step of intensity $I_{0}$ is

$I(s)=\frac{I_{0}}{s}$

The drop in voltage across the impedance in Fig. 3a after applying such a current step is

$V_{\mathrm{U}}(s)=I(s) Z(s)=\frac{I_{0}}{s}\left[R_{\mathrm{S}}+\frac{1}{A s^{\alpha}}\right]=\frac{I_{0} R_{\mathrm{S}}}{s}+\frac{I_{0}}{A s^{\alpha+1}}$ 
The corresponding inverse Laplace transform is

$v_{\mathrm{U}}(t)=L^{-1}\left\{V_{\mathrm{U}}(s)\right\}=L^{-1}\left\{\frac{I_{0} R_{\mathrm{S}}}{s}\right\}+L^{-1}\left\{\frac{I_{0}}{A s^{\alpha+1}}\right\}$

The inverse Laplace transform of the first term is

$L^{-1}\left\{\frac{1}{s}\right\} \leftrightarrow u(t)$

For the second term in (A.5) we have

$L^{-1}\left\{\frac{1}{s^{x}}\right\} \leftrightarrow\left(\frac{t^{x-1}}{\Gamma(x)}\right) u(t)$

Therefore, if we define

$x=\alpha+1$

then in (A.5) we obtain

$v_{\mathrm{U}}(t)=\left(I_{0} R_{\mathrm{S}}+\frac{I_{0}}{A} \frac{t^{\alpha}}{\Gamma(\alpha+1)}\right) u(t)$

Now, if we decompose the square-wave current as shown in Fig. 7, we have

$$
\begin{aligned}
i(t)= & I_{0}[0.5 u(t)-1.5 u(t-T)+2 u(t-2 T) \\
& -2 u(t-3 T)+\cdots]
\end{aligned}
$$

where $u(t)$ is the unit step and $f=1 /(2 T)$ is the signal frequency.

The intensities of the first and second steps are not $+I_{0}$ and $-2 I_{0}$ as it would correspond to a square wave of intensities $+I_{0}$ and $-I_{0}$. Because the constant-phase element is capacitive for $\alpha>0$, there is an integration effect (A.8) that for an applied current would result in a dc voltage drop across that element, thus leading to electrolysis in the electrodes (Fig. 8b). To avoid
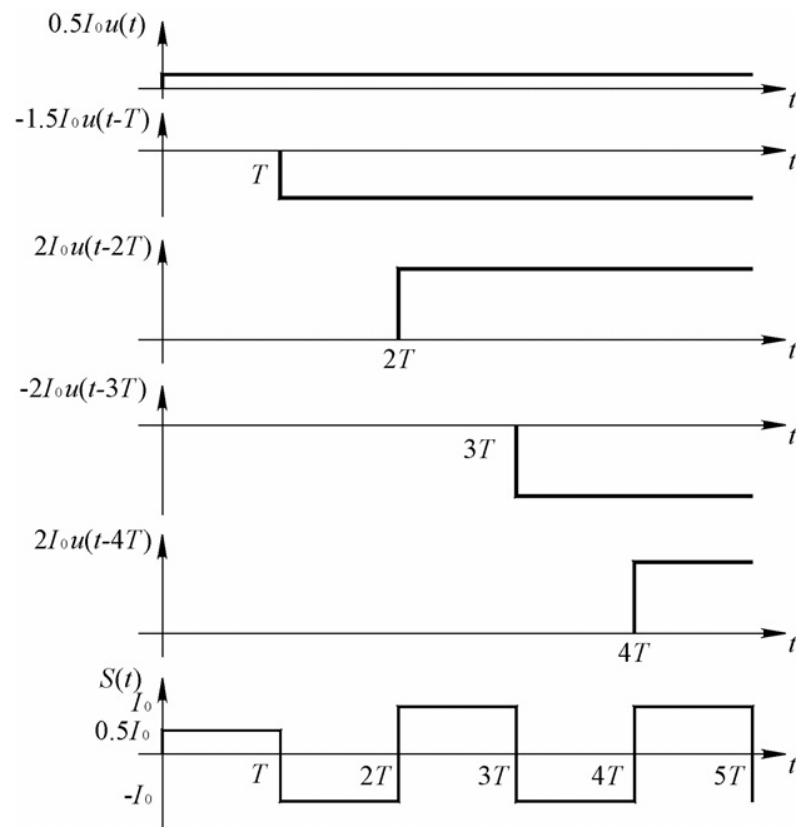

Fig. 7. A square-wave current $S(t)$ can be decomposed as a sum of step currents with amplitudes $0.5 I_{0},-1.5 I_{0}, 2 I_{0},-2 I_{0}, 2 I_{0}$ and so on, each step delayed by $M T$ seconds $(M=0,1,2, \ldots)$.

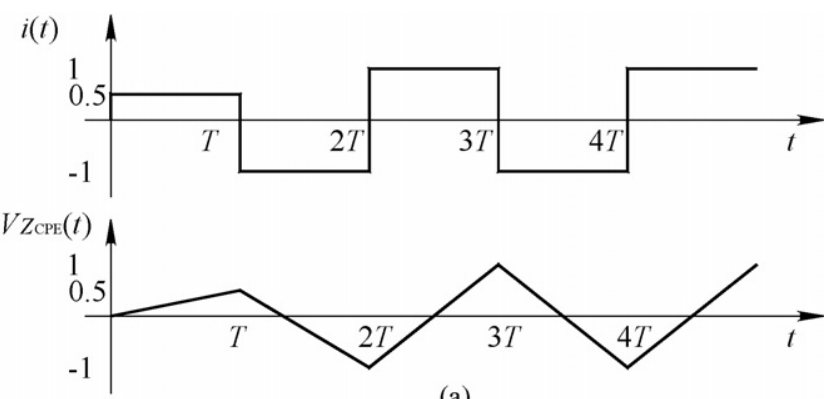

(a)
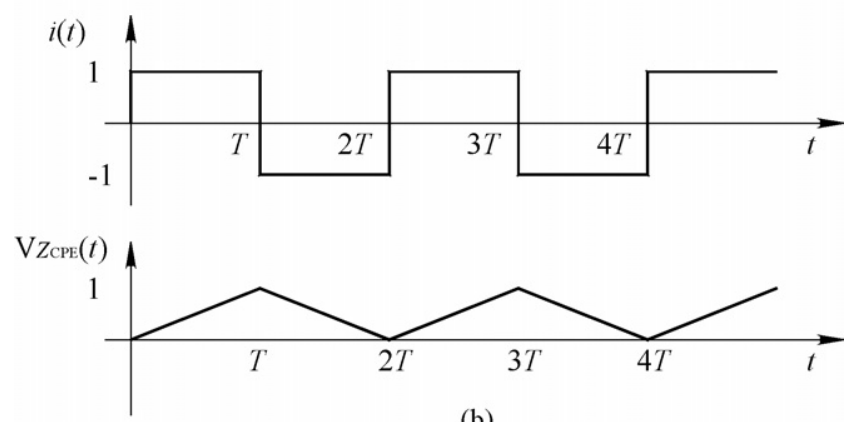

(b)

Fig. 8. (a) When the intensities of the currents steps are $+0.5 I_{0},-1.5 I_{0},+2 I_{0}$, $-2 I_{0},+2 I_{0}, \ldots$, the average drop in voltage across the constant-phase element is zero. (b) If the intensities of the current steps are $+I_{0},-2 I_{0},+2 I_{0},-2 I_{0}$, $+2 I_{0}, \ldots$, the average drop in voltage across the constant-phase element is not zero.

this effect, the intensity of the first step has been selected $0.5 I_{0}$ and that of the second step $-1.5 I_{0}$ (Fig. 8a). In both cases, $\alpha=1$. The voltage produced by the square-wave current in (A.9) is

$$
\begin{aligned}
v(t)= & 0.5 v_{\mathrm{U}}(t)-1.5 v_{\mathrm{U}}(t-T)+2 v_{\mathrm{U}}(t-2 T) \\
& -2 v_{\mathrm{U}}(t-3 T)+\cdots
\end{aligned}
$$

At any given instant, the voltage $v(t)$ will be the result of the voltages produced by the previous step currents. For $t$ such that $2 M T \leq t<(2 M+1) T$, where $M$ is any positive integer, we can consider $t=2 M T+m \Delta T$, where $\Delta T=T / N, N$ being the number of voltages measurements to perform each period plus 1 , and $m$ is any positive integer such that $0<m<N$. The voltage across $Z(s)$ at any instant $t$ is then

$$
\begin{aligned}
v(t)= & v(2 M T+m \Delta T)=0.5 v_{\mathrm{U}}(2 M T+m \Delta T) \\
& -1.5 v_{\mathrm{U}}(2 M T-T+m \Delta T) \\
& +2 v_{\mathrm{U}}(2 M T-2 T+m \Delta T) \\
& -2 \sum_{k=2}^{M} i_{\mathrm{U}}\{[2(M-k)+1] T+m \Delta T\} \\
& +2 \sum_{n=2}^{M} i_{\mathrm{U}}[2(M-n) T+m \Delta T]=V_{0}+V_{-}+V_{+}
\end{aligned}
$$

$V_{0}$ is the sum of the three voltages that correspond to the first three steps, whose amplitudes are $+0.5 I_{0},-1.5 I_{0}$ and $+2 I_{0}$. $V_{-}$is the sum of the voltages produced by all current steps whose amplitude is $-2 I_{0}$ (at $3 T, 5 T, \ldots$ ). $V_{+}$is the sum of the 
voltages produced by the current steps whose amplitude is $+2 I_{0}$ (at $4 T, 6 T, \ldots$ ). For $M \gg 1$, these three voltages can be written as, respectively

$$
\begin{aligned}
V_{0}= & 0.5 v_{\mathrm{U}}(2 M T+m \Delta T)-1.5 v_{\mathrm{U}}(2 M T-T+m \Delta T) \\
& +2 v_{\mathrm{U}}(2 M T-2 T+m \Delta T) \\
= & I_{0} R_{\mathrm{S}}+\frac{I_{0}}{A} \frac{1}{\Gamma(\alpha+1)}\left[0.5(2 M T+m \Delta T)^{\alpha}\right. \\
& \left.-1.5(2 M T-T+m \Delta T)^{\alpha}+2(2 M T-2 T+m \Delta T)^{\alpha}\right]
\end{aligned}
$$

$$
\begin{aligned}
V_{-} & =-2 \sum_{k=2}^{M} v_{\mathrm{U}}\{[2(M-k)+1] T+m \Delta T\} \\
& =-2 \sum_{k=2}^{M}\left[I_{0} R_{\mathrm{S}}+\frac{I_{0}}{A} \frac{\Delta T^{\alpha}}{\Gamma(\alpha+1)}\{[2(M-k)+1] N+m\}^{\alpha}\right]
\end{aligned}
$$

$$
\begin{aligned}
V_{+} & =+2 \sum_{n=2}^{M} v_{\mathrm{U}}[2(M-n) T+m \Delta T] \\
& =+2 \sum_{n=2}^{M}\left[I_{0} R_{\mathrm{S}}+\frac{I_{0}}{A} \frac{\Delta T^{\alpha}}{\Gamma(\alpha+1)}[2(M-n) N+m]^{\alpha}\right]
\end{aligned}
$$

Replacing (A.12)-(A.14) in (A.11) yields

$$
\begin{aligned}
& v(2 M T+m \Delta T)=V_{0}+V_{-}+V_{+} \\
& =I_{0} R_{\mathrm{S}}+\frac{I_{0}}{A} \frac{\Delta T^{\alpha}}{\Gamma(\alpha+1)}\left[0.5(2 M N+m)^{\alpha}\right. \\
& \quad-1.5(2 M N-N+m)^{\alpha}+2(2 M N-2 N+m)^{\alpha} \\
& \quad-2 \sum_{k=2}^{M}\{[2(M-k)+1] N+m\}^{\alpha} \\
& \left.+2 \sum_{n=2}^{M}[2(M-n) N+m]^{\alpha}\right]
\end{aligned}
$$

Simplifying this equation leads to

$v(2 M T+m \Delta T)=I_{0} R_{\mathrm{S}}+\frac{I_{0}}{A} \frac{\Delta T^{\alpha}}{\Gamma(\alpha+1)} \Psi_{m}(\alpha, M, N)$

where $\Psi_{m}(\alpha, M, N)$ is the sum of the contributions of the current steps previous to $t=2 M T+m \Delta T$,

$$
\begin{aligned}
& \Psi_{m}(\alpha, M, N) \\
& =0.5(2 M N+m)^{\alpha}-1.5(2 M N-N+m)^{\alpha} \\
& \quad+2(2 M N-2 N+m)^{\alpha}-2 \sum_{k=2}^{M}\{[2(M-k)+1] N+m\}^{\alpha} \\
& \quad+2 \sum_{n=2}^{M}[2(M-n) N+m]^{\alpha}
\end{aligned}
$$

The corresponding voltages $V_{1}, V_{2}$ and $V_{3}$ at times $T_{1}=2 M T+\Delta T, T_{2}=2 M T+2 \Delta T$ and $T_{3}=2 M T+3 \Delta T$ are

$$
\begin{aligned}
V_{1} & =v\left(T_{1}\right)=v(2 M T+\Delta T) \\
& =I_{0} R_{\mathrm{S}}+\frac{I_{0}}{A} \frac{\Delta T^{\alpha}}{\Gamma(\alpha+1)} \Psi_{1}(\alpha, M, N) \\
V_{2} & =v\left(T_{2}\right)=v(2 M T+2 \Delta T) \\
& =I_{0} R_{\mathrm{S}}+\frac{I_{0}}{A} \frac{\Delta T^{\alpha}}{\Gamma(\alpha+1)} \Psi_{2}(\alpha, M, N) \\
V_{3} & =v\left(T_{3}\right)=v(2 M T+3 \Delta T) \\
& =I_{0} R_{\mathrm{S}}+\frac{I_{0}}{A} \frac{\Delta T^{\alpha}}{\Gamma(\alpha+1)} \Psi_{3}(\alpha, M, N)
\end{aligned}
$$

In order to determine $\alpha$, we first calculate the voltage difference ratio

$\Phi(N)=\frac{V_{1}-V_{2}}{V_{2}-V_{3}}=\frac{\Psi_{1}(\alpha, M, N)-\Psi_{2}(\alpha, M, N)}{\Psi_{2}(\alpha, M, N)-\Psi_{3}(\alpha, M, N)}$

Then, the function that relates $\alpha$ to $\Phi(N)$ is determined by calculating $\Psi_{1}(\alpha, M, N), \Psi_{2}(\alpha, M, N), \Psi_{3}(\alpha, M, N)$ and $\Phi(N)$ for each $\alpha$ value between 0 and $1(0 \leq \alpha \leq 1)$. $\alpha$ is calculated from a curve fit procedure applied to the voltage ratios in (A.21). For $N=4$, we obtain

$\alpha=0.014954 \times \Phi^{2}(4)-1.5942 \Phi(4)+2.4501$

Once $\alpha$ is known, $A$ can be calculated from (A.18) and (A.19),

$V_{1}-V_{2}=\frac{I_{0}}{A} \frac{\Delta T^{\alpha}}{\Gamma(\alpha+1)}\left[\Psi_{1}(\alpha, M, N)-\Psi_{2}(\alpha, M, N)\right]$

Because $\Psi_{1}(\alpha, M, N)$ and $\Psi_{2}(\alpha, M, N)$ are known for the particular $\alpha$ value previously calculated, $A$ is

$A=\frac{I_{0}}{V_{1}-V_{2}} \frac{\Delta T^{\alpha}}{\Gamma(\alpha+1)}\left[\Psi_{1}(\alpha, M, N)-\Psi_{2}(\alpha, M, N)\right]$

The series resistance in (A.1) can be obtained by solving (A.18) for $R_{\mathrm{S}}$,

$R_{\mathrm{S}}=\frac{V_{1}}{I_{0}}-\frac{1}{A} \frac{\Delta T^{\alpha}}{\Gamma(\alpha+1)} \Psi_{1}(\alpha, M, N)$

where $\alpha$ and $A$ are those obtained in (A.22) and (A.23), respectively.

\section{References}

[1] S. Grimnes, Ø.G. Martinsen, Bioimpedance and Bioelectricity Basics, Academic Press, San Diego, 2000.

[2] J. Wu, J.P. Stark, A low-cost approach for measuring electrical conductivity and relative permittivity of liquids by triangular waveform voltage at low frequencies, Meas. Sci. Technol. 16 (2005) 1234-1240.

[3] B. Carkhuff, R. Cain, Corrosion sensors for concrete bridges, IEEE Instrum. Meas. Mag. 6 (2003) 19-24.

[4] L.A. Geddes, Historical evolution of circuits models for the electrode-electrolyte interface, Ann. Biomed. Eng. (1997) 1-14. 
[5] L.A. Geddes, R. Roeder, Measurement of the direct-current (Faradic) resistance of the electrode-electrolyte interface for commonly used electrode materials, Ann. Biomed. Eng. 29 (2001) 181-186.

[6] J. Ross Macdonald, Impedance Spectroscopy, Wiley, New York, 1987.

[7] B. Rigaud, J.P. Morucci, N. Chauveau, Bioelectrical Impedance Techniques in Medicine. Part I: Bioimpedance Measurement, Second Section: Impedance Spectroscopy, Begell House, New York, Crit. Rev. Biomed. Eng. 24 (1996) 257-351.

[8] R. Pallàs-Areny, J.G. Webster, Sensors and Signal Conditioning, second ed., Wiley, New York, 2001.

[9] J. Lario-García, R. Pallàs-Areny, Measurement of three independent components in impedance sensors using a single square wave, Sens. Actuators A-Phys. 110 (2004) 164-170.

[10] S. Westerlund, L. Ekstam, Capacitor theory, IEEE Trans. Dielectr. Electr. Insulation 1 (5) (1994) 826-839.

[11] A.K. Jonscher, The 'universal' dielectric response, Nature 267 (1977) 673-679.

\section{Biographies}

Javier Lario-García received the Telecommunication Engineer degree from the Technical University of Catalonia (UPC), Barcelona, Spain, in 1995. He has a 10year experience in industrial design of medical and analytical instrumentation.
$\mathrm{He}$ is currently a PhD candidate at the Department of Electronic Engineering at the same University. His research work is about electrical impedance analysis based on digital signals, particularly applied to conductivity measurements in electrolytes and bioimpedance measurements.

Ramon Pallàs-Areny received the Ingeniero Industrial and Doctor Ingeniero Industrial degrees from the Technical University of Catalonia (UPC), Barcelona, Spain, in 1975 and 1982, respectively. He is a professor of electronic engineering at the same University, and teaches courses in medical and electronic instrumentation. In 1989 and 1990 he was a visiting Fulbright Scholar and, in 1997 and 1998, he was an Honorary Fellow at the University of Wisconsin, Madison. In 2001, he was nominated Professor Honoris Causa by the Faculty of Electrical Engineering of the University of Cluj-Napoca (Romania). His research includes instrumentation methods and sensors based on electrical impedance measurements, sensor interfaces, non-invasive physiological measurements and electromagnetic compatibility in electronic systems. He is the author and coauthor of several books on instrumentation in Spanish, Catalan and English, the latest being Sensors and Signal Conditioning, second ed. (Wiley, New York, 2001) and Analog Signal Processing (Wiley, New York, 1999), with John G. Webster, and Direct Sensor-to-Microcontroller Interfaces (Marcombo, Barcelona, 2005). Dr. Pallàs-Areny was a recipient, with John G. Webster, of the 1991 Andrew R. Chi Prize Paper Award from the Instrumentation and Measurement Society (IEEE). He is a Fellow of the IEEE and a member of the International Society for Measurement and Control (ISA). 